Knie J. 2021 · 3:149-150

https://doi.org/10.1007/s43205-021-00114-y Angenommen: 9. Juni 2021

Online publiziert: 19. Juli 2021

C Springer Medizin Verlag $\mathrm{GmbH}$, ein Teil von Springer Nature 2021

\title{
One Minute Wonder
}

\section{Schneller und effizienter Wissenserwerb zwischendurch!}

\author{
Wolf Petersen \\ Klinik für Orthopädie und Unfallchirurgie, Martin-Luther-Krankenhaus Berlin, Berlin, Deutschland
}

Neue Erkenntnisse und Daten verhalten sich gerade in der Medizin sehr dynamisch. Daher ist es unabdingbar, sich ständig mit neuen Inhalten auseinanderzusetzen. Der Transfer von neuen Kenntnissen aus der Wissenschaft in die reale Patientenversorgung ist dabei jedoch eine Herausforderung [2]. Gerade in Zeiten von zunehmender Arbeitsverdichtung stoßen daher neue Methoden der Wissensvermittlung im medizinischen Alltag auf großes Interesse [4, 5].

In anderen medizinischen Disziplinen wie z.B. der Pflege, in der Dermatologie oder der Intensiv- und Notfallmedizin hat sich in den letzten Jahren das aus England stammende Konzept der "One Minute Wonder" (OMW) als neue effektive Fortbildungsmethode etabliert [1, 3, 7, 8].

\section{॥ Die Ideen sollten aus der betreffenden Berufsgruppe selbst kommen - aus der Praxis für die Praxis}

Ein OMW besteht aus einer Lerntafel, deren Inhalt in einer Minute aufgenommen werden kann. Dabei sollen regelmäßig wiederkehrende Wartezeiten innerhalb der Arbeitszeit (z. B. OP-Aufenthaltsraum, Arztzimmer, Kopierer etc.) zur Wissensvermittlung und Auffrischung von Wissen genutzt werden. Das oberste Ziel eines OMW ist es, Wissen und Informationen einfach, schnell und zielgerichtet mit vielen Kollegen und Kolleginnen zu teilen.

Die Ideen sollten, sofern möglich, aus der betreffenden Berufsgruppe selbst kommen - aus der Praxis für die Praxis. Durch ihre knappe und ansprechende Darstellung sind die OMW besonders geeignet, um sie z.B. auch in Arztzimmern, an Druckern oder in Pausenräumen aufzu- hängen und kurze Wartezeiten während der Arbeit sinnvoll zur Fortbildung zu nutzen.

Dieses visuell ausgerichtete Lernkonzept scheint auch für die Orthopädie und Unfallchirurgie interessant zu sein. Daher möchten wir das „One Minute Wonder" als neue Rubrik für das Knie Journal einführen. Es soll Ziel sein, in jeder Ausgabe des Knie Journal ein OMW zu publizieren. Das erste OMW in diesem Heft befasst sich mit unserem CME-Thema aus dem letzten Heft "Quadrizepssehnenruptur" [6]. Es behandelt die Diagnostik und Therapie dieser seltenen Sehnenruptur. Es soll als Vorlage für weitere OMW dienen. Dabei orientiert sich dieses OMW stark am bereits publizierten CME-Beitrag.

Wir hoffen, dass wir Sie, die Autoren und Leser des Knie Journal für dieses neue Format begeistern können. Dabei müssen sich die OMW nicht unbedingt an einem konkreten (CME-)Beitrag orientieren, um dieses Thema zu vertiefen. Im OMW könne auch Teilaspekte des Beitrages herausgestellt werden (z. B. Diagnostik oder ei-

\section{Infobox 1}

Empfehlungen zum Erstellen eines One Minute Wonder (OMW)

- Ein OMW sollte innerhalb einer Minute gelesen und aufgenommen werden können

- Konzentration auf wenige Aspekte

- Kurze Aufzählungen, Checklisten, schrittweises Vorgehen

- Keine langen Texte

- Beachtung der Grundsätze des Plagiats und des Urheberrechts

- Nutzung von Symbolen, Bildern und Grafiken

- Nutzung von QR-Codes zur Verlinkung auf weitere Informationen (z. B. Artikel im Knie Journal, Video etc.) 
ne bestimmte Methode). Das Thema des OMW kann auch losgelöst von einem Artikel sein. Wichtiger als die Anlehnung an einen bestehenden Beitrag ist, dass das Thema des OMW auch zu diesem Format passt.

Wir hoffen daher auf weitere Vorschläge aus dem Leser- und Autorenkreis. Auch für die Komitees der Deutschen Kniegesellschaft wäre die Erstellung eines OMW eine schöne Initiative. $\bullet$ Infobox 1 gibt eine Übersicht über die Empfehlungen zum Erstellen eines OMW. Am wichtigsten ist bei der Erstellung eines OMW, dass sich die Autoren auf Informationen konzentrieren, die innerhalb einer Minute aufgenommen werden können.

Wir hoffen, dass wir mit diesem Format die Fortbildungslandschaft in Orthopädie und Unfallchirurgie bereichern können und dass es bei unseren Leserinnen und Lesern auf Interesse stößt. Das OMW soll bisherige Fortbildungsmethoden nicht ersetzen, sondern sinnvoll ergänzen.

\section{Korrespondenzadresse}

\section{Prof. Dr. med. Wolf Petersen}

Klinik für Orthopädie und Unfallchirurgie,

Martin-Luther-Krankenhaus Berlin

Caspar-Theyß-Str. 27-31, 14193 Berlin,

Deutschland

wolf.petersen@jsd.de

Interessenkonflikt. W. Petersen hat Beraterhonorare der Firmen Karl Storz und Deustches Instituts für Zell und Gewebetransplantation (DIZG) des sowie Vortragshonorare der Firmen Otto Bock Health Care, Plasmaconcept, OPED, Stryker und Geistlich erhalten.

\section{Literatur}

1. Eppler F, Monninger M, Schempf B et al (2019) One Minute Wonder - Fachwissen to go. Notfall Rettungsmed 22:642-644. https://doi.org/10. 1007/s10049-019-00639-w

2. Jahed J, Bengel J, Baumeister H (2012) Transfer von Forschungsergebnissen in diemedizinische Praxis. Gesundheitswesen 74(11):754-761. https://doi. org/10.1055/s-0031-1286277

3. Kind J, Zink A (2021) One Minute Wonder ergänzende, effiziente Wissensvermittlung auf einen Blick. Hautarzt 72(1):42. https://doi.org/10. 1007/s00105-020-04741-w

4. Petersen W, Voss A (2021) Innovationen in der Ausbildung. Arthroskopie 34:93. https://doi.org/ 10.1007/s00142-021-00443-0

5. Petersen W, Hees T, Ohde J (2021) Aktueller Stand virtueller Simulatoren für die chirurgische Ausbildung in Orthopädie und Unfallchirurgie. Arthroskopie 34:94-102. https://doi.org/10.1007/ s00142-020-00429-4
6. Petersen W, Bierke S, Häner M (2021) Quadrizepssehnenruptur. Knie J. https://doi.org/10.1007/ s43205-021-00108-w

7. Rowlinson J (2014) The one minute wonder network. Clin Teach 11(5):332-335. https://doi. org/10.1111/tct.12160

8. Schmidt B, Krüger L (2016) Lernen in nur einer Minute. Intensivpflege 24(05):258-259. https:// doi.org/10.1055/s-0042-110559

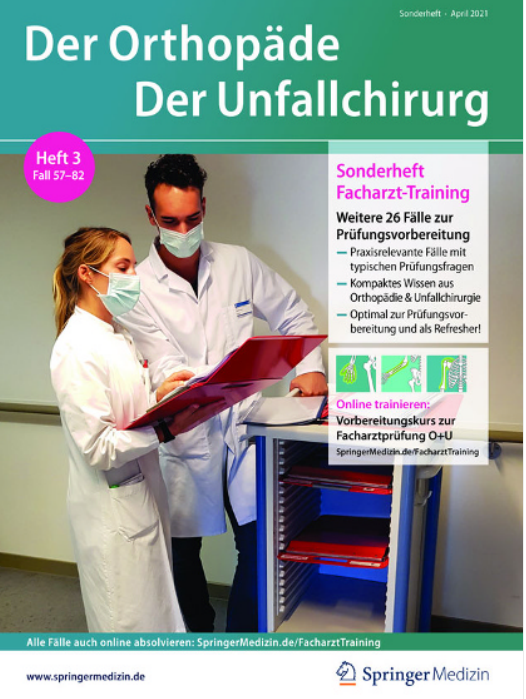

\section{Machen Sie sich fit mit dem Facharzt-Training Orthopä- die \& Unfallchirurgie!}

Bereiten Sie sich auf die Facharztprüfung vor oder möchten Sie Ihr Fachwissen mit typischen Fallbeispielen aus der Orthopädie \& Unfallchirurgie auffrischen? Dann sind die 3 Sonderhefte Facharzt-Training Orthopädie \& Unfallchirurgie von Der Orthopäde und Der Unfallchirurg genau das Richtige für Sie!

Sie finden in diesen Heften:

- praxisnahe Fallbeispiele, systematisch und aktuell aufbereitet

- mit Prüfungsfragen und Antworten

- Kompaktes Wissen aus Orthopädie \& Unfallchirurgie

- Insgesamt 3 Hefte decken in 82 Fällen exemplarisch alle wichtigen Themen der Facharztprüfung ab

- Von Expert*innen für Sie geplant, geschrieben und begutachtet

Weitere Informationen sowie zwei Beiträge zum Problesen finden Sie unter www.springermedizin.de/sonderheftou.

Bestellen Sie sich das Facharzt-Training O\&U nach Hause:

- je Einzelheft $44 €$

- Paketpreis für alle 3 Hefte: $99 €$ unter o.g. Link oder unter Angabe des Aktionscodes C0019580 per E-Mail bei:

Marie-Luise.Witschel@springer.com 